\title{
Fast target detection method for high-resolution SAR images based on variance weighted information entropy
}

\author{
Zongjie Cao*, Yuchen Ge and Jilan Feng
}

\begin{abstract}
Since the traditional CFAR algorithm is not suitable for high-resolution target detection of synthetic aperture radar (SAR) images, a new two-stage target detection method based on variance weighted information entropy is proposed in this paper. On the first stage, the regions of interest (ROls) in SAR image is extracted based on the variance weighted information entropy (WIE), which has been proved to be a simple and effective quantitative description index for the complex degree of infrared image background. Considering that SAR images are nonuniform, an experiment is conducted ahead, in which the value of the variance WIE from a real SAR image in three areas with significant different uniform levels are tested and compared. The results preliminarily verified that the variance WIE is able to measure the complex degree of SAR images. After that, in order to make the segmentation efficient, the rough ROls are further processed with a series of methods which adjust ROls into regular pieces. On the second stage, for each of the ROls, a variational segmentation algorithm based on the Split-Bregman algorithm is adopted to extract the target. In our experiment, the proposed method is tested on two kinds of SAR images, and its effectiveness is successfully demonstrated.
\end{abstract}

Keywords: Synthetic aperture radar; Target detection; Variance weighted information entropy; Variational segmentation

\section{Introduction}

Man-made objects can be divided into linear targets and blob targets. For linear targets, like roads, railways, bridges, and airport runway, they often appear to have obvious characteristics of straight line or curve and can be described approximately through an accurate extraction of the line feature. However, blob targets like tanks, ships, vehicles, aircraft, bunkers, oil depots, power plants, and other types of construction usually have a reunion in the spatial distribution, which means that the targets are located in a rectangular area in the image as a whole.

This paper is focused on the detection of blob targets.

Among applications of synthetic aperture radar (SAR) images, the automatic target recognition (ATR) system is of great importance. The target detection of SAR images, serving as the first stage of ATR systems, provides a basis for the validity of subsequent recognition.

By now, the most widely used target detection algorithm is the constant false alarm detection (CFAR).

* Correspondence: zjcao@uestc.edu.cn

School of Electronic Engineering, UESTC, Chengdu 611731, China
CFAR algorithms detect targets by pixel-wise threshold operation, in which the threshold is adaptively computed for each pixel according to the statistical property of pixels within a local window [1-7].

However, since CFAR is a kind of pixel level methods of target detection, it is not suitable for SAR images of high resolution in reality. First of all, the serious speckle impact of high-resolution SAR image increases the probability of false alarm. Secondly, in high-resolution SAR images, a target often occupies a lot of resolution cells and is with multiple number of independent strong scattering. After CFAR detection, a target is likely to be composed of a plurality of distributed pixels that cannot form a connected region, which increases the probability of false alarm and the loss of information of structure and shape.

To overcome the problems of traditional CFAR algorithm, this paper proposes a target extraction algorithm of high-resolution SAR image based on 'location-segmentation' stages and a new detection character. As is known, traditional one-dimensional graph entropy is a 
statistical form of characteristics which depicts the accumulation characteristics of gray level distribution and reflects the average information of a figure. However, it can only represent global information rather than the property of its spatial relationships [8,9]. To optimize this character, a derivative named the variance weighted information entropy (WIE) was applied firstly in the detection of infrared images. Due to the fact that infrared objects with different radiation usually appear to have distinct gray value in real images, the variance WIE has been proved to be a simple and effective quantitative description index for the complex degree of infrared image background [10]. Analogous to the infrared images, the SAR images are also nonuniform. Hence, in this paper, the value of the variance WIE from a real SAR image in its uniform area, area including ups and downs, and the area which contains the target is primarily tested. The result preliminarily verifies that the values of highly nonuniform areas which allude to regions of interest (ROIs) are significantly greater than that of homogeneous backgrounds.

In the first stage, ROIs are obtained through an iterative algorithm based on WIE and averaging segmentation. This means allowed us to roughly and quickly locate several potential targets. However, due to their restrictions, targets are often not completely included in the ROI or are not completely at the center of the ROI. To get an effective segmentation in the next stages, this paper proposes a series of processing methods subsequently, including false alarm exclusion algorithm, rectangular-completing algorithm of ROI, and centroid alignment algorithm to refine the ROIs and filter out false alarms. On the second stage, the variational SAR image segmentation method is utilized to get ROI pieces. This target segmentation method of variational model under the framework of partial differential can be both flexible and robust, expressing the boundary region naturally and integrating a variety of image information organically to complete the image segmentation. For extracting SAR target segmentation based on partial differential equation, it mainly involves two aspects: establishing a reasonable energy functional to describe SAR image characteristics and finding effective optimization methods of the energy functional. In the experiment part, this new algorithm is tested with results that confirm the validity in both high-resolution SAR images in real and composed SAR images with transcendental position information.

The rest of the paper is organized as follows. Section 2 introduces the location stage of the high-resolution SAR images based on the variance weighted information entropy. In Section 3, the segmentation stage and the consolidated flowchart of the whole algorithm are shown. Section 4 is devoted to experimental results for SAR target extraction with the proposed techniques. Conclusions are drawn in Section 5.

\section{Positioning}

In this section, the variance WIE is introduced and a small experiment is conducted so as to preliminarily prove that the variance WIE could measure the complex degree of SAR images. After that, the flowchart of the entire location stage and the detailed steps are introduced respectively.

\subsection{The variance weighted information entropy}

The gray value of pixels in SAR Images can reflect spatial distribution of the energy emitted by electromagnetic waves. Accordingly, the concept of entropy in information theory is extended in the field of image processing. Serving as a derivative application of information entropy proposed by Shannon, graph entropy is a statistical form of characteristics, which reflects the average information of a figure.

For a digital image of $M$ rows and $N$ columns with a gray level $i 0<i \leq 255$, after getting its probability distribution of each gray level, let $f_{i}$ be the observed frequencies of gray level $i$, then the occurrence probability of gray scale $i$ is as follows:

$$
P_{i}=\frac{f_{i}}{M \times N}, i \in[0,255] \quad \sum_{i=0}^{255} f_{i}=M \times N
$$

and the one-dimensional graph entropy can be expressed as

$$
H=-\sum_{i=0}^{255} p_{i} \log p_{i}
$$

when $P_{i}=0$, let $P_{i} \log P_{i}=0$.

By minimizing $H$, we can get $p_{i}=1, i \in[0,255]$ so that the digital image only has a certain gray level which is $i$. This gives a uniform distribution.

By analyzing its nature further, several conclusions can be drawn as follows:

1. Graph entropy can reflect the degree of difference in the gray values of pixels. With a smaller value of graph entropy, the spatial distribution of energy within the window is more uniform and the difference in the gray values of pixels is weaker. When the gray values of all pixels within the window are the same, the graph entropy reaches the minimum that is $H=0$. On the contrary, when the entropy value is greater, the difference in the gray values of pixels within the window is big due to the existence of blob targets.

2. The calculation of graph entropy is a kind of nonlinear spatial filtering. When blob targets appeared, the mutation of gray value in a local region can lead to a mutation of graph entropy since the background of the SAR image is rather 
homogeneous or slowly varying and the background noise can be fitted by a certain random noise model with similar entropy.

3. Being less sensitive to the imaging sensor and geometric distortion, graph entropy can help to locate potential target area correctly.

Although one-dimensional graph entropy can depict the accumulation characteristics of gray level distribution, it can only represent global information rather than the property of its spatial relationships $[8,9]$. Hence, the variance WIE is introduced in this paper to deal with location processing.

The variance WIE was first applied to the detection of infrared images. Due to the fact that infrared objects with different radiation usually appear to have distinct gray value in real images, the variance WIE which has been proved to be a simple and effective quantitative description index for the complex degree of infrared image background [10] is introduced.

For a subimage extracted from the original with a gray level $i(0<i \leq 255)$,

$$
H(i)=-\sum_{i=0}^{255}(i-\bar{i})^{2} P_{i} \log P_{i}
$$

When $P_{i}=0$, let $P_{i} \log P_{i}=0$, where $P_{i}$ is the probability of the gray levels in the infrared image, and $\bar{i}$ is the mean intensity of the infrared image.

Equation 3 shows that the impact of pixels with gray levels $i$ on the weighted entropy can be reflected by a certain weight. By defining it into $(i-\bar{i})^{2}$, pixels that are significantly different from the local average can be given a big influence on the entropy, and the local area with potential target can be better highlighted.

Considering that SAR images are also nonuniform and that graph entropy is insensitive to the imaging sensor and geometric distortion, in this paper, WIE is imported to the detection stage of SAR images.

Analogous to the infrared image, the variance WIE of SAR images can be expressed as Equation 3 as well, but with the denotations of $P_{i}$ revised as the probability of the gray levels in the SAR image and $P_{i}$ as the mean intensity of the SAR image.

To verify the conjecture, the value of WIE from a real SAR image in its uniform area, area including the ups and downs, and area which contains the target is tested. The result is shown in Figure 1.

\subsection{Target extraction by a location-segmentation strategy} Briefly, this average WIE algorithm based on 'positioning-segmentation' is reflected in a 'rough-fine' structure: First of all, extract blocks pieces which may contain the target through ROIs [11]. Then, refine the ROIs and conduct the variational segmentation. In fact, the rough extraction process involves multiple iteration and image updating. The accurate ROIs are gained through continuous reciprocating operational steps in the local part too.

As mentioned before, one-dimensional graph entropy can only represent global information rather than the property of its spatial relationships. In order to obtain the target position information, the SAR image is divided into pieces, using both global WIE as well as local WIE to reflect the information.

Here is the consolidated flowchart of the whole algorithm (Figure 2).

\subsubsection{Pretreatment}

Firstly, measure the size of the input image $F$ and denote it by $X \times Y$ Due to the fact that an averaging segmentation method is proposed to the subsequent stage of ROI rough extractions which demands for the size of the image, the image boundary needs to be intercepted or expanded.

Suppose the closest power of two items for $X$ is $2^{M}$ and closest power of two items for $Y$ is $2^{N}$, then the image boundary is intercepted or expanded into $2^{M} \times 2^{N}$. After that, the global WIE from the pretreated image $F$ is got through formula (3).

According to the conjecture of SAR images preliminarily verified before, areas which contain a target have a higher WIE value, so the SAR image is divided into blocks with certain size. Serving as potential blocks for ROIs, the seed block can be gained through getting its local WIE and a given threshold. Particularly, in the ROI detection algorithm, the idea of ROI iteration is introduced

For images that contain multiple objectives, in order to get the complete ROIs through iteration, a global threshold $T h$ is needed to be set as follows:

$$
T h=k \times E(H)
$$

where $E(H)$ is the mean of the variance WIE values of the whole image, and $k$ is a constant which defines the number of detected target. An inappropriate value of $k$ will cause the phenomenon of false alarm or missed alarm. The experience range of $k$ is $[0.5,3]$.

\subsubsection{Rough extraction}

After pretreatment, the size of the image $F$ has been adjusted to $2^{M} \times 2^{N}$ in the following steps:

Step 1 Evenly divide image $F$ into four subregions of $2^{M-1} \times 2^{N-1}$ and label them (Figure 3 ).

Step 2 For subregions from 1 to 4 , calculate their WIE value respectively. 


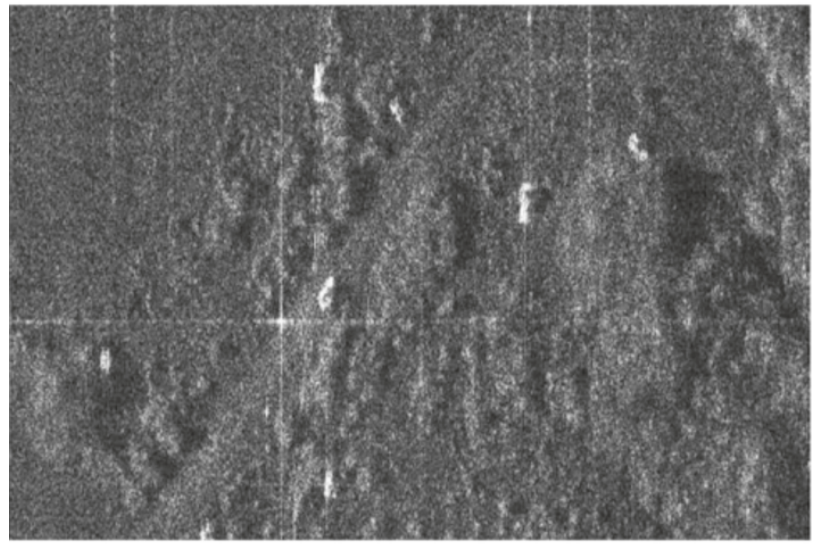

(a)

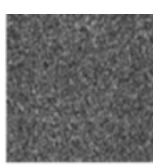

(b)

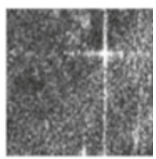

(c)

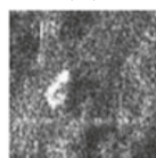

(d)

Figure 1 The WIE value of a real SAR image in different areas. (a) The original image. (b) A uniform area, WIE $=0.58$. (c) An area including the ups and downs, WIE $=0.76$. (d) An area which contains the target, WIE $=0.82$. The sizes of $(\mathbf{b}, \mathbf{c}, \mathbf{d})$ are all $100 \times 100$ pixels.

Step 3 Label the subregion with maximum WIE value and denote its value into $H_{\max }$.

Step 4 Get the mean $\mu$ and the standard deviation $\sigma$ of the labeled subregion in step 3, so the subthreshold $H t$ of it is measured as follows:

$$
H t=\mu+\alpha \times \sigma
$$

where $\alpha$ is a constant which defines the size of the seed block. If the value of $\alpha$ is too small, it may result in too small sized ROIs, which cannot contain the entire targets, and a too big $\alpha$ may lead to too big sized ROIs, which contains too much background. The range of $\alpha$ is set as $[0.5,3]$.

Step 5 Judge whether $H_{\max }$ is greater than $H t$, if $H_{\max } \geq$ $H t$, then the labeled area in step 3 is a seed block; if $H_{\text {max }}<H t$, let $m=m / 2, n=n / 2$ and repeat step 1 to step 4 until the maximal value in the variance weighted information SAR image satisfies $H_{\max } \geq H t, m=2$, or $n=2$.

The effect of iteration is similar to the following (Figure 4):

Step 6 Rough extraction of ROI - judge the seed block attended by step 5 again; if $H_{\max }>T h$, the seed block is truly a ROI and then go to step 7; otherwise, the ROI detection stops.

Step 7 Update image - the gray values of the pixels in the ROI are set to the mean gray value, and then go back step 1 using the updated image.

\subsubsection{ROI adjustment}

The averaging segmentation based on mean WIE can roughly determine the location of ROIs; however, due to their restrictions, targets are often not completely included in the ROI or are not completely at the center of the ROI. To get an effective segmentation in the next stages, this paper proposes a series of processing methods, including false alarm exclusion algorithm, rectangular-completing algorithm of ROI, and centroid alignment algorithm to adjust the ROI.

First of all, a false alarm exclusion algorithm based on the knowledge of target size is applied to all ROIs in the image. Among ROIs, whose area is less than the setting value, level will be ruled out.

Next, a rectangular-completing algorithm of ROIs is conducted, adjusting ROI with irregular shape to a rectangle (Figure 5).

Finally, let the geometric center of the ROIs be aligned with the mass center of the corresponding area on the image $F$.

In a two-dimensional space, the coordinate formula of the mass center is expressed particularly as

$$
r_{m}=\frac{\sum m_{i} r_{i}}{m_{i}}
$$

where $\mathrm{r} \in[x, y] ; m_{i}$ denotes the weighting of pixel $i$; and $r_{i}$ denotes the coordinate of pixel $i$;

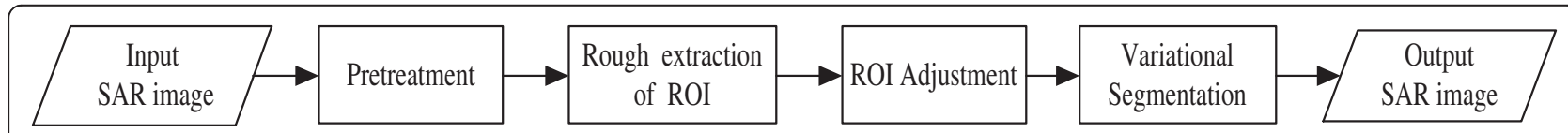

Figure 2 Consolidated flowchart of the whole algorithm. 


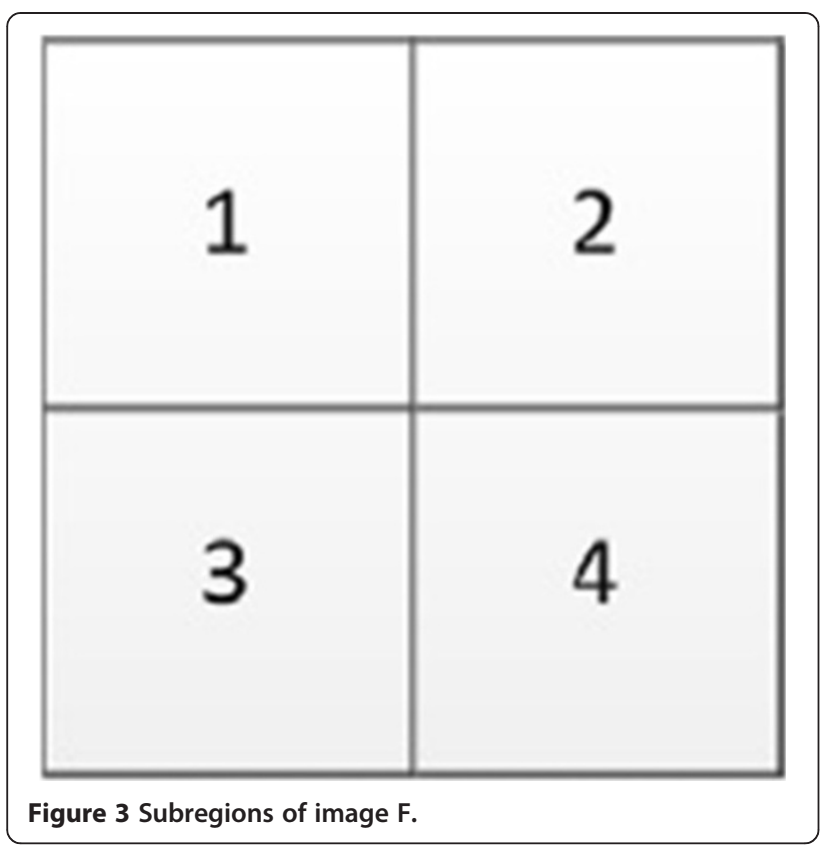

Let $r_{g}$ be the geometric center of a piece of ROI, then the displacement distance $v$ is as follows:

$$
v=r_{m}-r_{g}
$$

Repeat the steps above until that $v$ is less than a certain standard (two units for instance).

\section{Segmentation}

When ROIs are selected and adjusted, targets are extracted from ROIs with image segmentation approach. Due to the impact of speckle noise, SAR image segmentation is known to be a difficult problem. In this paper, this problem is addressed with a variational SAR image segmentation approach which mainly involves two aspects: establishing a reasonable energy functional to describe SAR image characteristics and finding effective optimization methods of the energy functional [12].

\subsection{Energy functional}

The definition of the energy functional is the key point to the level set segmentation, which shall be able to express the characteristic of SAR images properly and reflect the differences between target and background. So when the target and the background are separated, the energy functional could reach the minimum value [12]. For variational segmentation based on partial differential equation, its energy functional has the general form as follows:

$$
E=E_{\mathrm{D}}+\lambda E_{\mathrm{P}}
$$

where $E_{\mathrm{D}}$ is the energy term driven by the characteristic of SAR images which is usually defined through regional probability statistics or texture information of the SAR images, and $E_{\mathrm{P}}$ is the regular energy term based on $a$ priori constraint information $[13,14]$.

In the framework of probability and statistics, assume that the characteristic of the two areas within a ROI under segmentation obeys the probability density function: $p_{i}\left(I(\boldsymbol{x}) ; \boldsymbol{\theta}_{i}\right)$ respectively. Let $I(x): \Omega \rightarrow R$ be the intensity SAR image, where $\Omega \subset R^{2}$ is the image domain. $i \in\{T, B\}$ represents either target or background. $\boldsymbol{\theta}_{i}, i=$ $1, \ldots, N$ is the parameter vector of distribution. Meanwhile, define the regional information functions to be $r_{i}\left(\boldsymbol{x} ; \Omega_{i}\right)=-\log p_{i}\left(I(\boldsymbol{x}) ; \boldsymbol{\theta}_{i}\right), i=1, \ldots, N$ so that the regional energy term shall be [15].

$$
\begin{aligned}
E_{\mathrm{D}} & =-\sum_{i \in\{T, B\}} \beta_{i} \int_{\Omega_{i}} \log p_{i}\left(I(\boldsymbol{x}) ; \boldsymbol{\theta}_{i}\right) d \boldsymbol{x} \\
& =\sum_{i \in\{T, B\}}^{N} \beta_{i} \int_{\Omega_{i}} r_{i}\left(\boldsymbol{x} ; \Omega_{i}\right) d \boldsymbol{x}
\end{aligned}
$$

By selecting different probabilistic models, different regional energy items can be defined and distinct SAR image segmentation methods can be gotten as well. In this paper, $G^{0}$ distribution is adopted $[15,16]$ so that the regional information function can be defined as
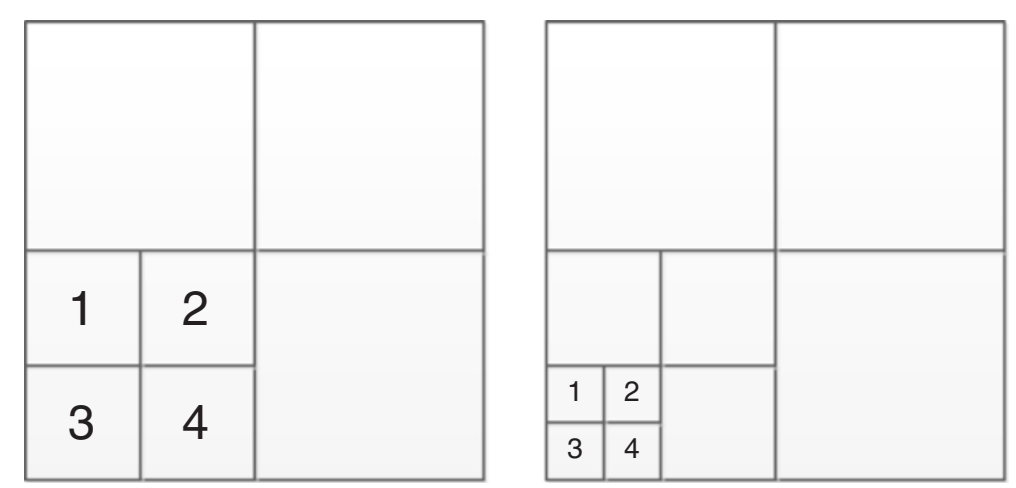

Figure 4 The schematic diagram of iteration. 


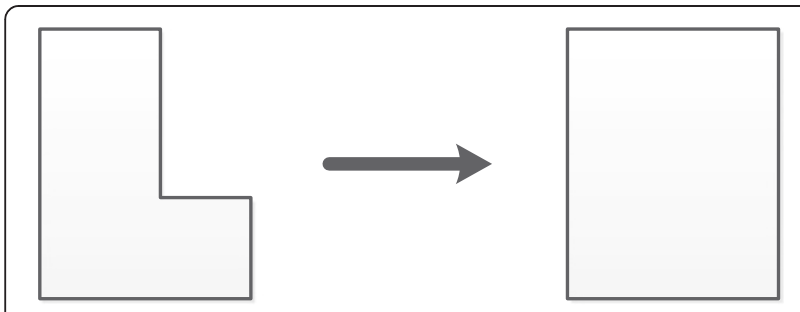

Figure 5 The schematic diagram of rectangular completing.

$$
\begin{aligned}
r_{i}^{G^{0}}(\boldsymbol{x})= & -\log p_{i}^{G^{0}}\left(\boldsymbol{x} ; \alpha_{i}, \gamma_{i}, L_{i}\right)=-\log \left(\frac{L_{i}^{L_{i}} \Gamma\left(L_{i}-\alpha_{i}\right)}{\gamma_{i}^{\alpha_{i}} \Gamma\left(L_{i}\right) \Gamma\left(-\alpha_{i}\right)}\right) \\
& -\left(L_{i}-1\right) \log (I(\boldsymbol{x})), i=1, \ldots, N+\left(L_{i}-\alpha_{i}\right) \log \left(\gamma_{i}+L_{i} I(\boldsymbol{x})\right)
\end{aligned}
$$

As for $E_{P}$ a common definition of it is the length of the boundary curve $C$ :

$$
E_{\mathrm{P}}=\int_{C} d s
$$

where $d s$ is the unit arc length, which if minimized, the split curve can been smoothed, small isolated areas can be eliminated, and boundary information could also been brought in. The boundary indicator function is defined as follows:

$$
e(\boldsymbol{x})=\frac{1}{1+\left|\nabla\left(G_{\sigma} * I(\boldsymbol{x})\right)\right|}
$$

where $G_{\sigma}$ is a Gaussian kernel function, convolving with $I$ to enhance the robustness of edge detection against noise. Also, the boundary energy can be defined through other edge detection algorithms, for instance, algorithms based on ratio operator, ROEWA operator, and so on. So the energy term can be defined as follows:

$$
E_{\mathrm{P}}=\int_{C} e_{C_{i}}(s) d s
$$

where $e_{C_{i}}(s)$ represents the value of $e(x)$ on the boundary curve $C_{i}$. When the active curve moves towards the edge, both the accuracy of edge location and the smoothness of the curve are achieved by importing the edge information.

With energy terms based on region and edge information, the energy functional of the segmentation of ROIs can be get as follows:

$$
E=\sum_{i \in\{T, B\}} \beta_{i} \int_{\Omega_{i}} r_{i}\left(\boldsymbol{x} ; \Omega_{i}\right) d \boldsymbol{x}+\int_{C} e_{C_{i}}(\boldsymbol{x}) d s
$$

\subsection{Minimization of the energy functional}

Usually, an appropriate choice to minimize the predefined energy functional of SAR image segmentation is necessary. In this paper, two means are adopted: level set means and fast optimization methods based on dual solution.

By bringing level set function $\phi_{i}: \Omega \rightarrow R, i=1, \ldots, N$ in formula (14), different areas can be represented as

$$
\left\{\begin{array}{lll}
\phi(x)>0 & \text { if } & x \in \Omega_{i} \\
\phi(x)<0 & \text { if } & \boldsymbol{x} \notin \Omega_{i} \\
\phi(x)=0 & \text { if } & \boldsymbol{x} \in C_{i}
\end{array}\right.
$$

with Heaviside function $H\left(\phi_{i}\right)$ and Dirac function $\delta\left(\phi_{i}\right)$ :

$$
\begin{aligned}
H(z) & =\frac{1}{2}\left[1+\frac{2}{\pi} \arctan \left(\frac{z}{\varepsilon}\right)\right], \delta(z)=H^{\prime}(z) \\
& =\frac{1}{\pi} \frac{\varepsilon}{\varepsilon^{2}+z^{2}}
\end{aligned}
$$

If $\phi_{i}>0$, then $H\left(\phi_{i}\right) \approx 1$; otherwise, if $\phi_{i}<0$, then $H$ $\left(\phi_{i}\right) \approx 0$, where $\varepsilon$ is usually a constant with small value to control the speed for $H\left(\phi_{i}\right)$ to change from 0 to 1 . With no information of a priori probabilities, the energy function can be represented as

$$
\begin{aligned}
E= & \sum_{i \in\{T, B\}} \int_{\Omega} H\left(\phi_{i}\right) r_{i}\left(\boldsymbol{x} ; \Omega_{i}\right) d \boldsymbol{x} \\
& +\sum_{i=1}^{N} \lambda_{i} \int_{\Omega} e(\boldsymbol{x})\left|\nabla H\left(\phi_{i}\right)\right| d \boldsymbol{x}
\end{aligned}
$$

If distribution parameters $\theta_{i}, i=1, \ldots, N$ are determined according to variational principle $\frac{\partial \phi_{i}}{\partial t}=-\frac{\partial E}{\partial \phi_{i}}$, the curve evolution equation can be get which makes the energy functional minimal:

$$
\begin{aligned}
\frac{\partial \phi_{i}}{\partial t}(\boldsymbol{x})= & \delta\left(\phi_{i}(\boldsymbol{x})\right) \\
& \times\left(\lambda \operatorname{div}\left(e(\boldsymbol{x}) \frac{\nabla \phi_{i}(\boldsymbol{x})}{\left|\nabla \phi_{i}(\boldsymbol{x})\right|}\right)+r_{i}(\boldsymbol{x})\right)
\end{aligned}
$$

Let $n$ be the number of iterations and $\tau$ be the step of time, then the iteration of level set function can be

$$
\phi_{i}^{n+1}(\boldsymbol{x})=\phi_{i}^{n}(\boldsymbol{x})+\tau \frac{\partial \phi_{i}^{n}}{\partial t}(\boldsymbol{x})
$$

By selecting different probabilistic models, different regional energy items can be defined and distinct SAR image segmentation methods can be gotten as well.

Although level set segmentation can get a satisfactory result, its evolution speed is relatively slow. To promote the efficiency of the segmentation of SAR images and enhance the real-time property of the system, this paper does further research on iteration method based on Split-Bregman algorithm $[17,18]$.

Since the energy function in (17) is nonconvex, the level set evolution equation in (18) is likely to fall into local minima, which brings about erroneous segmentation results. 
So to achieve a fast SAR image segmentation globally, the energy function of SAR image segmentation shall be designed as a convex function with a global minimum. For the level set evolution equation in (19), the termination condition of evolution is as follows:

$$
\delta\left(\phi_{i}\right)\left(\alpha \operatorname{div}\left(e_{C_{i}}(x) \frac{\nabla \phi_{i}}{\left|\nabla \phi_{i}\right|}\right)+r_{i}(x)\right)=0
$$

Formula (20) can be seen as the minimization condition of the convex energy functional:

$$
E_{G}\left(x ; u_{i}\right)=\alpha \int_{\Omega} e_{C_{i}}(x)\left|\nabla u_{i}\right| d x+\int_{\Omega}\left[r_{i}(x) u_{i}\right] d x, u_{i} \in[0,1]
$$

The energy functional in (21) is convex, with global minimum. Because formula (20) and (18) are actually the same conditions, the minimum of (14) can be get by solving the minimum in (21), which, in the meantime, can be proved to be equivalent to the following minimization problem:

$$
\min _{u_{i} \in[0,1]} \int_{\Omega}\left(e_{C_{i}}(x)\left|\nabla u_{i}\right|+\lambda e_{i}(x) u_{i}\right) d x
$$

It is hard to solve for the partial differential, because in formula (22), it has both the level set function $u_{i}$ and its first order differential form $\left|\nabla u_{i}\right|$. To achieve quickly, let $\rightarrow d_{i}=\nabla u_{i}$ so formula (22) can be transformed into the following constraint minimization problem:

$$
\min _{u_{i} \in[0,1]} \int_{\Omega}\left(e_{C_{i}}(x)||_{i} \mid+\lambda e_{i}(x) u_{i}\right) d x \text { sub } \stackrel{\mathrm{V}}{d}_{i}=\nabla u_{i}
$$

With Lagrange multiplier method, the problem in (23) can be transformed into the following unconstrained minimization problem:

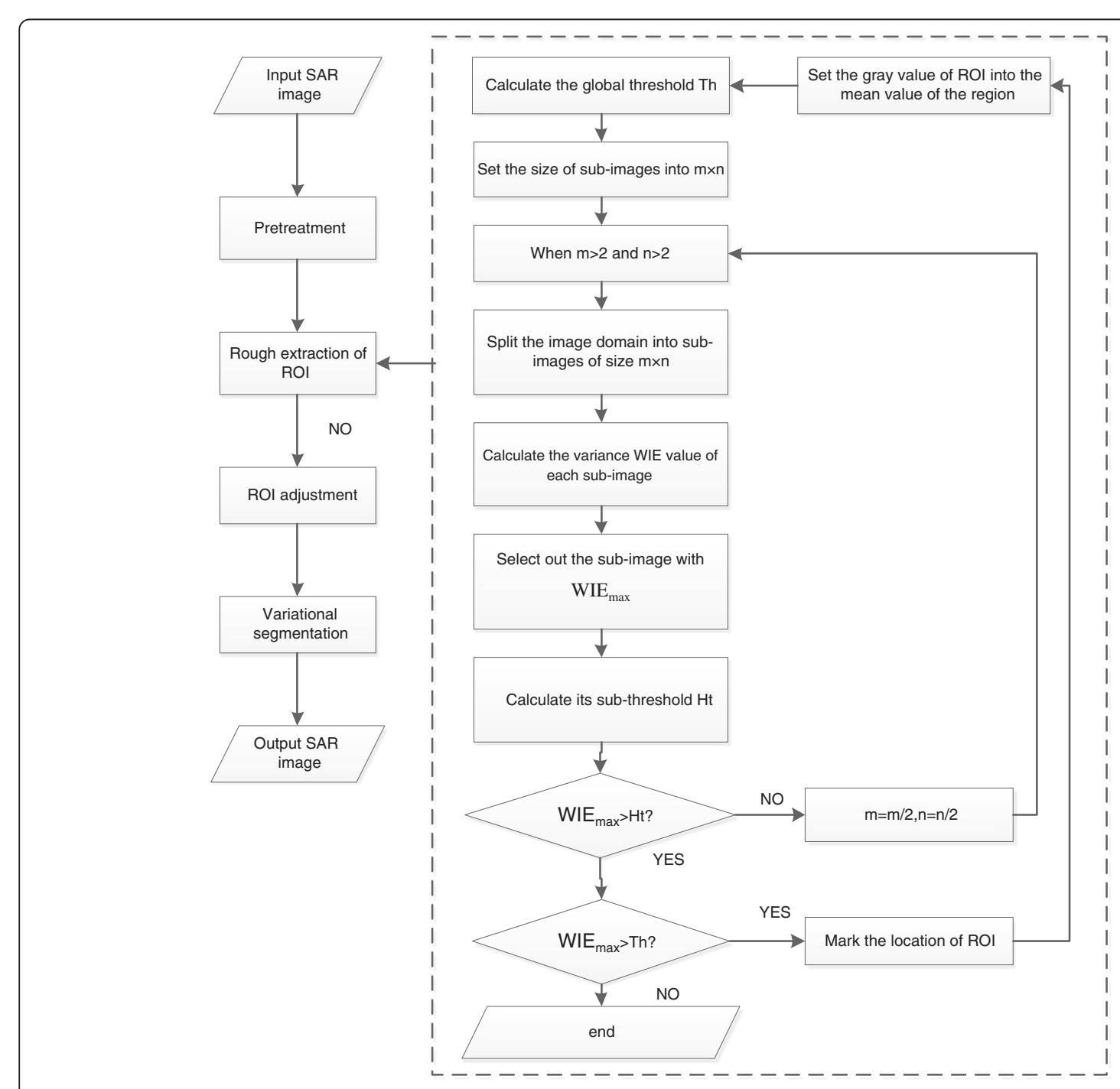

Figure 6 Architecture of the proposed fast target detection method of high-resolution SAR image based on location-segmentation stages. 


$$
\min _{u_{i} \in[0,1]} \int_{\Omega}\left(e_{C_{i}}(x)\left|d_{i}\right|+\lambda e_{i}(x) u_{i}\right)+\frac{\eta}{2}\left\|\left.\right|_{i}-\nabla u_{i}\right\|_{2}^{2} d x
$$

It can be proved that the problem in (24) could be solved through the iteration method of Bregman by introducing the error vector $\stackrel{\mathrm{V}}{b_{i}}=\stackrel{\mathrm{V}}{d_{i}}-\nabla u_{i}$ :

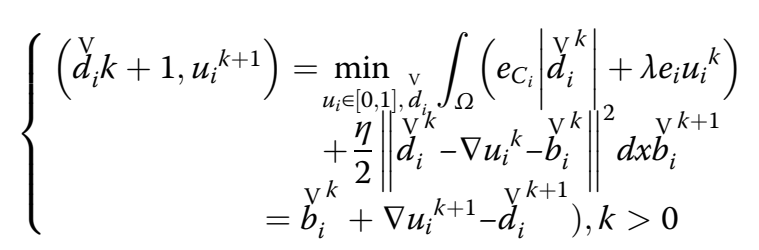

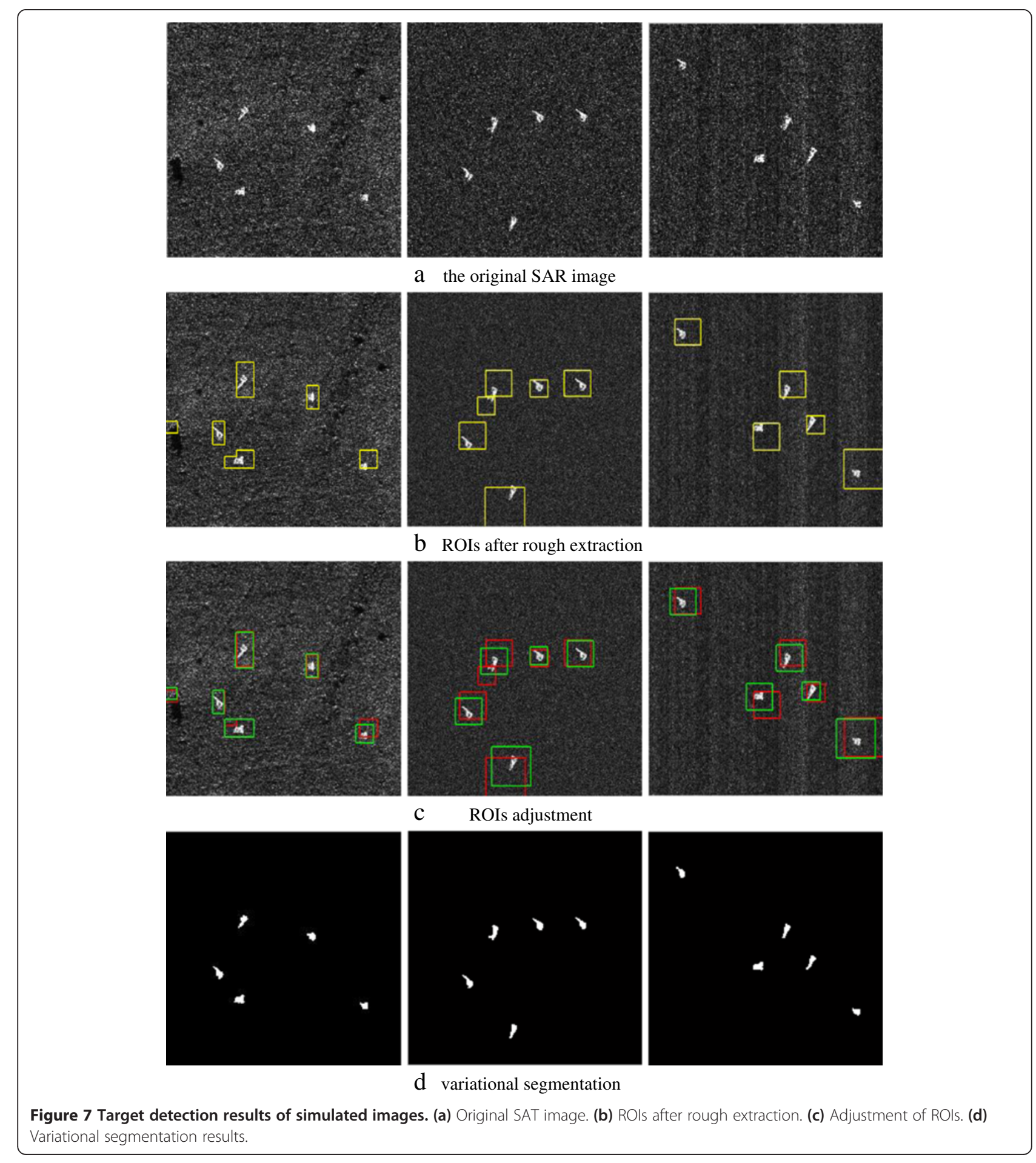


The first equation above can be solved in two steps. Firstly, get the variational calculus of $u$ and make it equal to 0 :

$$
\eta \Delta u_{i}^{k+1}=\lambda e_{i}+\eta \operatorname{div}\left(\begin{array}{c}
\mathrm{v}^{k} \\
b_{i} \\
\mathrm{v}_{i}^{k}
\end{array}\right), u_{i} \in[0,1]
$$

Discretize the Laplacian operator and divergence operator as follows:

$$
\begin{aligned}
& \Delta u_{s, t}^{i}=u_{s+1, t}^{i}+u_{s, t+1}^{i}+u_{s-1, t}^{i}+u_{s, t-1}^{i}-4 u_{s, t}^{i} \\
& \operatorname{div}\left(\mathrm{V}^{i}\right)_{s, t}=p_{s-1, t}^{V_{i x}}-\underline{p}_{s, t}^{i x}+p_{s, t-1}^{V_{i y}^{i y}}-p_{s, t}^{V_{i y}}
\end{aligned}
$$

where $\stackrel{v^{i x}}{p^{i y}}$ are two components of the twodimensional vector $\mathrm{p}^{i}$. In order to distinguish between themselves, let $u^{i}=u_{i}$ and solve (26) through the iteration of Gauss-Seidel:

$$
\begin{aligned}
& u u_{s, t}^{k+1}=\frac{u_{s-1 . t}^{i k}+u_{s+1 . t}^{i k}+u_{s, t-1}^{i k}+u_{s, t+1}^{i k}-\operatorname{div}\left(b_{i}^{k}\right)_{s, t}+\operatorname{div}\left(\check{d}_{i}^{k}\right)_{s, t}-\frac{\lambda}{\eta} e_{i, s, t}}{4} \\
& u_{i}^{k+1}=\max \left(\min \left(u u^{k+1}, 1\right), 0\right)
\end{aligned}
$$

where the second expression above is to meet the conditions that $u_{i} \in[0,1]$. To solve the minimization problem for $\dot{d}_{i}^{k}$ in the first expression, we have

$$
\begin{aligned}
& {\stackrel{\mathrm{y}}{d_{i}}}^{k+1}=\operatorname{shrink}_{e_{c_{i}}}\left({\stackrel{\mathrm{v}}{b_{i}}}^{k}+\nabla u_{i}{ }^{k+1}, \eta\right) \\
& =\frac{b_{i}^{\mathrm{v}^{k}}+\nabla u_{i}^{k+1}}{\left|\mathrm{v}_{i}^{k}+\nabla u_{i}^{k+1}\right|} \max \left(\left|b_{i}^{\mathrm{v}}+\nabla u_{i}^{k+1}\right|-\eta^{-1} e_{C_{i}}, 0\right)
\end{aligned}
$$

So the iterative calculation of (25), (28), and (29) can achieve the purpose of fast solution of the minimization of (14) and the segmentation of ROI.

\subsection{The overall process}

In summary, the architecture of the proposed fast target detection method of high-resolution SAR image based on location-segmentation stages is shown in Figure 6.

\section{Experiments and results}

Figure 7 shows a group of synthetic images. Due to the fact that the positions of the targets are already known, it is used to the test algorithm. Figure $7 \mathrm{a}$ shows three simulated SAR images which contain five different targets by using the MSTAR measured data respectively. After extracting ROIs from the SAR image based on the variance WIE on first stage, the location results are shown in Figure $7 \mathrm{~b}$. It can be seen that the method achieves comparatively good positioning of the target area. Nevertheless, the irregular shape of the ROIs and the fact that some targets are lying at the boundary of the ROIs are not conducive to subsequent processing.

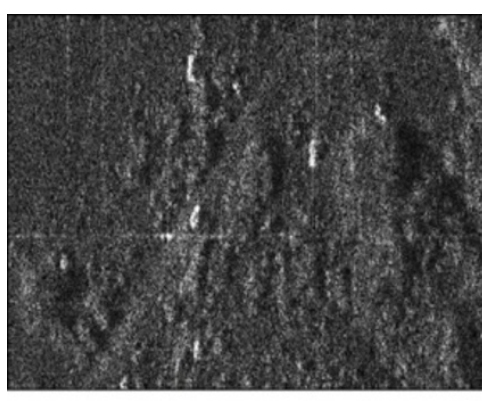

a the original SAR image

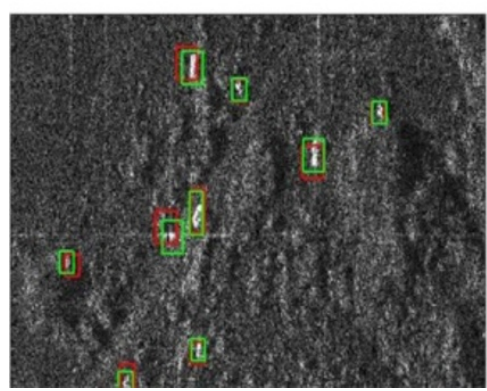

C ROIs adjustment

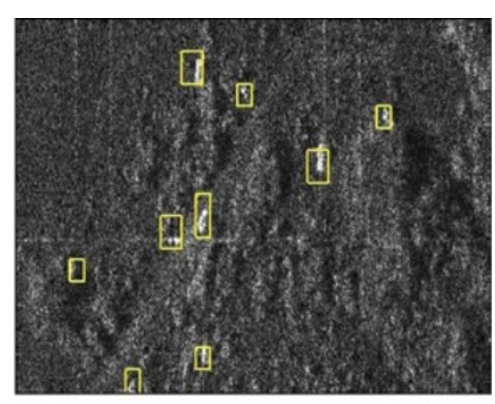

b ROIs after rough extraction

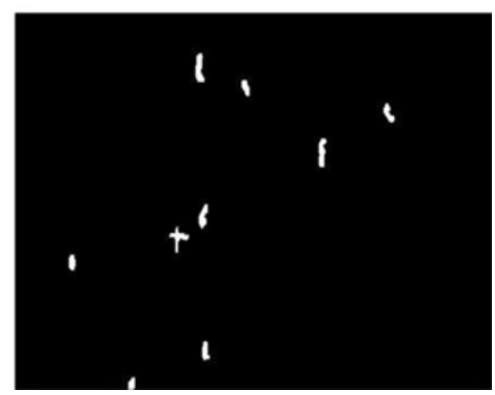

d variational segmentation

Figure 8 Target detection results of real SAR image. (a) Original SAR image. (b) ROls after rough extraction. (c) Adjustment of ROls. (d) Variational segmentation results. 
Hence, the rectangular-completing algorithm of ROI and centroid alignment algorithm area conducted to refine the ROI region. The results in Figure $7 \mathrm{c}$ include two types of lines which are red and green; the former type shows the outline of original ROIs without any further processing, and the latter one sketches the outline of ROIs after the rectangular-completing stages and the centroid alignment stages which confirm that the target areas are getting better positions. For the extracted ROIs, a segmentation algorithm based on variational model is adopted to accurately extract the target. The results are shown in
Figure $7 \mathrm{~d}$ with all targets preferably positioned and extracted and the shape information remains relatively intact.

Figure 8 shows a group of tanks in the wild which contains trees, grass, road, tanks, military facilities, and other complex objects types. Figure 8a defines the original SAR image of $512 \times 1,024$ pixels. The yellow lines in Figure $8 \mathrm{~b}$ sketch the outline of ROI that has deformity shapes after rough extraction. Figure $8 \mathrm{c}$ includes two types of lines which are red and green; the former type shows the outline of ROIs after the rectangular-completing stages, and the latter one sketches the outline of ROIs after the

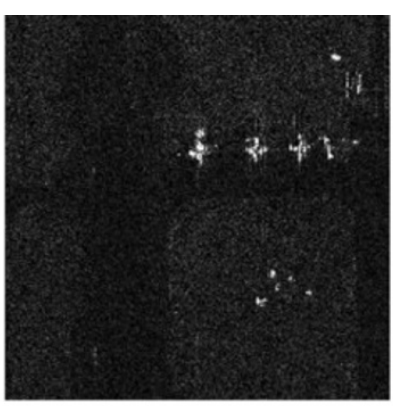

(a) the original SAR image

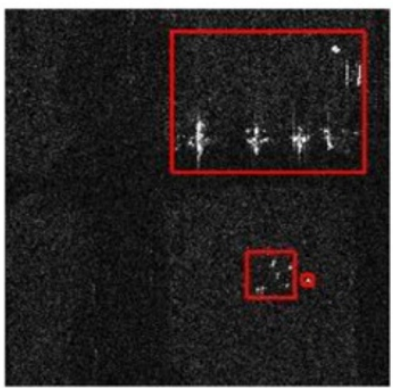

(c) ROIs adjustment

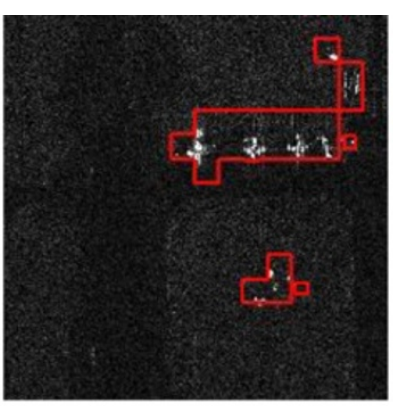

(b) ROIs after rough extraction

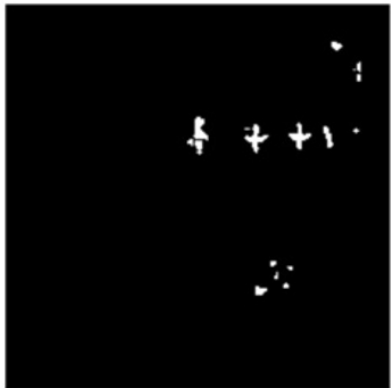

(d) variational segmentation
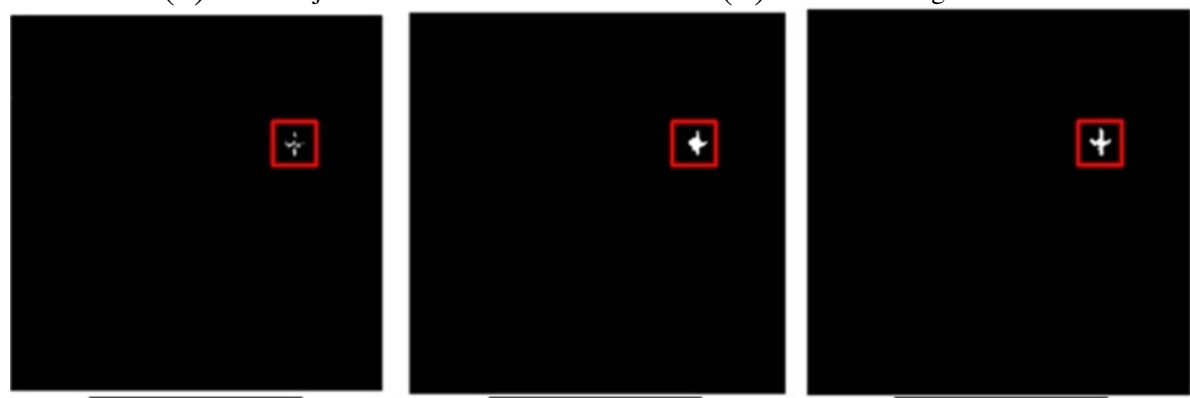

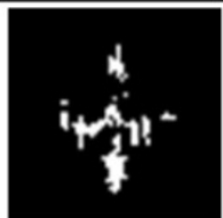

(e) traditional CFAR method

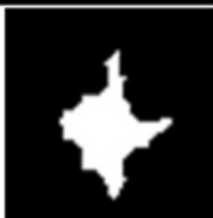

(f) CFAR+ morphological operation

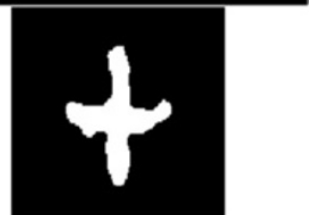

(g) two-stage target detection method

Figure 9 Comparison between the proposed methods and the traditional CFAR methods. (a) Original SAR image. (b) ROls after rough extraction. (c) Adjustment of ROls. (d) Variational segmentation results. (e) Traditional CFAR methods. (f) CFAR with morphological operation. (g) Two-stage target detection method. 
centroid alignment stages. The obvious changes between Figure $8 \mathrm{~b}$ and Figure $8 \mathrm{c}$ verified the effectiveness of ROI adjustment. The last picture in Figure 8 shows the output image after variational segmentation. The whole program takes $6.93 \mathrm{~s}$ in this picture.

In order to further illustrate the superiority of this proposed method in Figure 9, a comparison between it and the traditional CFAR method is shown. For the details in a specific object, it can be seen clearly in Figure 9e that the result from the traditional CFAR algorithm is incomplete with some parts of a same object been detected disconnectedly. Although these discrete parts can get connected by conducting morphological operation in Figure 9f, the shape distortion of the target is rather serious. Figure $9 \mathrm{~g}$ shows the detection result from the proposed method with idea extraction of the ROIs, and the shape of the targets is comparatively complete with accurate shape information.

\section{Conclusions}

This paper proposed a novel two-stage target detection method for high-resolution SAR images. Since the variance WIE characteristic has been preliminarily verified to measure the complex degree of the SAR images, the defects for traditional CFAR algorithm to conduct detection for SAR images with high resolution have been overcome. Besides, the adjustment stage which includes three parts guaranteed the precise segmentation of ROIs consequently. Moreover, the variational segmentation stage gave rise to a better outcome compared with traditional level set segmentation method by running faster and giving more accurate result. The experiment in Section 4 verified the effectiveness of our algorithm.

\section{Abbreviations}

ATR: automatic target recognition; CFAR: constant false alarm rate; ML: mean level; ROls: regions of interest; SAR: synthetic aperture radar; WIE: variance weighted information entropy.

\section{Competing interests}

The authors declare that they have no competing interests.

\section{Acknowledgement}

This work was supported in part by the National Natural Science Foundation of China under Projects 60802065, 61271287 and 61371048.

Received: 7 January 2014 Accepted: 14 March 2014 Published: 3 April 2014

\section{References}

1. HM Finn, RS Johnson, Adaptive detection mode with threshold control as a function of spatially sampled clutter level estimates. RCA Rev. 29(3), 414-464 (1968)

2. M Barkat, SD Himonas, PK Varshney, CFAR detection for multiple target situations. IEE Proc. Radar and Signal Processing F. 136(5), 193-209 (1989)

3. H Rohling, Radar CFAR thresholding in clutter and multiple target situations. IEEE Trans. on AES 19(3), 608-621 (1983)

4. CJ Kim, DS Han, HS Lee, Generalized OS CFAR detector with no coherent integration. Signal Process. 31(1), 43-56 (1993)

5. DT Nagle, J Saniie, Performance analysis of linearly combined order statistic CFAR detectors. IEEE Trans. On AES 31(2), 522-533 (1995)
6. I Ozgunes, PP Gandhi, SA Kassam, A variably trimmed mean CFAR radar detector. IEEE. Trans. On AES 28(4), 1002-1014 (1992)

7. H Goldman, Performance of the excision CFAR detector in the presence of interferers. IEE Proc. Radar and Signal Processing F. 137(3), 163-171 (1990)

8. L Yang, J Yang, Adaptive detection for infrared small target under sea-sky complex background. Electronics. Let. 40(17), 1083-1085 (2004)

9. L Yang, J Yang, J Ling, New criterion to evaluate the complex degree of sea-sky infrared background. Optical Engineering. 44(12), 126401-126406 (2005)

10. L Yang, Y Zhou, J Yang, L Chen, Variance WIE based infrared images processing. Electron. Let. 42(15), 857-859 (2006)

11. Y Li, X Mao, D Feng, Y Zhang, Fast and accuracy extraction of infrared target based on Markov random field. J. Signal Process. 91(5), 1216-1223 (2011)

12. ZJ Cao, LL Pang, YM Pi, A variational level set approach for automatic target extraction of SAR images, in 1st Asian and Pacific Conference on Synthetic Aperture Radar, APSAR 2007 (IEEE, Piscataway, 2007), pp. 375-378

13. ZJ Cao, YM Pi, XB Yang, JT Xiong, A variational level set SAR image segmentation approach based on statistical model, in 2008 7th European Conference on Synthetic Aperture Radar (EUSAR). Friedrichshafen, 2-5 June 2008, pp. 1-4

14. ZJ Cao, R Rui, LL Pang, YM Pi, A variational level set SAR image segmentation approach based on statistical model. J. Electron. Inform. Technol. 12, 017 (2008)

15. JL Feng, ZJ Cao, YM Pi, A level set approach for SAR image segmentation based on G(0) distribution. Modern Radar 32(12), 35-39 (2010)

16. JL Feng, ZJ Cao, YM Pi, Variational SAR image segmentation based on the G0 model and an augmented Lagrangian method. Progress Electromagnet. Res. B. 39, 373-392 (2012)

17. T Goldstein, S Osher, The split Bregman method for L1-regularized problems. SIAM J. Imaging Sci. 2(2), 323-343 (2009)

18. T Goldstein, X Bresson, S Osher, Geometric applications of the split Bregman method: segmentation and surface reconstruction. J. Sci. Comput. 45(1-3), 272-293 (2010)

doi:10.1186/1687-6180-2014-45

Cite this article as: Cao et al:: Fast target detection method for

high-resolution SAR images based on variance weighted information entropy. EURASIP Journal on Advances in Signal Processing 2014 2014:45.

\section{Submit your manuscript to a SpringerOpen ${ }^{\circ}$ journal and benefit from:}

- Convenient online submission

- Rigorous peer review

- Immediate publication on acceptance

- Open access: articles freely available online

- High visibility within the field

- Retaining the copyright to your article

Submit your next manuscript at $>$ springeropen.com 